\title{
Synthesis and characterization of $\gamma$-glycine $-\mathbf{a}$ nonlinear optical single crystal for optoelectronic and photonic applications
}

\author{
A. Arputha latha ${ }^{1}$, M. Anbuchezhiyan ${ }^{2, *}$, C. Charles Kanakam ${ }^{3}$, K. Selvarani $^{4}$ \\ ${ }^{1}$ Department of Physics, Prince Shri Venkateshwara Padmavathy Engineering College, Kanchipuram Dt., India \\ ${ }^{2}$ Department of Physics, Valliammai Engineering College, SRM Nagar, Kattankulathur - 603 203, India \\ ${ }^{3}$ Former Professor, Department of Chemistry, Presidency College, Chennai, India \\ ${ }^{4}$ Department of Physics, Sri Sairam Engineering College, Kanchipuram Dt., India
}

\begin{abstract}
A single crystal of gamma-glycine (GG), a polymorph of glycine, was synthesized by crystallization. The single crystal of GG was grown from an aqueous solution. The morphology of GG was studied in order to assess its growth facets. The good quality single crystals were subjected to X-ray diffraction studies to reveal their structure. The FT-IR spectral analysis was carried out to confirm the presence of expected functional groups. The UV-Vis analysis was done for GG single crystals to determine the optical transparency and band gap. Simultaneous TG-DTA analysis was employed to understand the thermal and physicochemical stability of the title compound. The mechanical stability and laser stability of GG single crystal were studied using Vickers microhardness test and laser induced damage threshold on different planes of the crystal to reveal its anisotropic nature. The dielectric measurement was carried out as a function of frequency and the results were discussed. The existence of second harmonic generation (SHG) of the title compound was confirmed by Kurtz-Perry powder technique. The SHG effective nonlinearity and particle size dependence of GG powder sample were compared with a standard reference material: potassium dihydrogen phosphate (KDP).
\end{abstract}

Keywords: single crystal; X-ray diffraction; optical properties; electrical properties; thermal properties; mechanical properties; phase transition

(C) Wroclaw University of Science and Technology.

\section{Introduction}

The nonlinear optics (NLO) is the field that includes all phenomena in which optical parameters of materials are changed by the interaction of intense coherent source of light. The NLO phenomena have led to the enhancement in understanding of light-matter interactions. The search for new molecular materials with NLO properties is currently the subject of considerable importance investigations due to their potential applications in photonic devices [1]. Family of amino acid crystals has been intensively studied by researchers for their excellent nonlinear optical properties. Glycine $\left(\mathrm{C}_{2} \mathrm{H}_{5} \mathrm{O}_{2} \mathrm{~N}\right)$ possesses the lowest molecular weight among all amino acids. Glycine, a white crystalline

\footnotetext{
*E-mail: 1lathu2005@gmail.com
}

amino acid, is found as a constituent of many proteins. It is a derivative of acetic acid. It is also known as aminoacetic acid. It is the simplest one among twenty natural amino acids, representing approximately one-third of collagen compositions. It has been widely used in crystal engineering. Unlike other amino acids, it has no asymmetric carbon and it is optically inactive. That is, it does not have D- and L-stereoisomer. It is the only protein forming amino acid without a center of chirality. It exists as zwitterions in solution and in a solid state. It is known that glycine, the simplest amino acid, exhibits three different polymeric forms viz. $\alpha$-glycine, $\beta$-glycine and $\gamma$-glycine. It is also a well-known fact that $\alpha$ - and $\beta$-glycine crystallizes in centrosymmetric space group $\mathrm{P} 2_{1} / \mathrm{c}$, thereby ruling out the basic condition for optical second harmonic generation. However, $\gamma$-glycine crystallizes 
in non-centrosymmetric space group $\mathrm{P} 3_{1}$ or $\mathrm{P} 3_{2}$ which makes it ideal for piezoelectric and NLO applications. The phenomenon of chemical species having more than one possible crystal form is known as a polymorphism. The ability of a molecule to exist in more than one solid-state structure is a result of differences in the molecular packing arrangement. The polymorphism may exist in any compound, but the conditions and methods required to prepare certain polymorphs have been still challenging. The control and optimization of the polymorphic form play a key role in the crystallization process, especially in the pharmaceutical industry. Polymorphic crystallization is affected by various factors, such as cooling rate, supersaturation, agitation, solvent, $\mathrm{pH}$ values, additives, solution concentration, impurity and seeding, etc. [2]. In the past years, many authors have reported the growth and various characterizations of $\gamma$-glycine with various additives, such as phosphoric acid, potassium chloride, potassium fluoride, lithium sulphate, lithium acetate and lithium nitrate [3-6]. Gamma glycine is currently grown by many conventional methods, such as slow cooling, slow evaporation as well as gel method. Though there are numerous reports on this compound, the extraordinary characteristics of gamma glycine force the researches to perform deeper studies on its properties. The main aim of this work is to explore and shed light into anisotropic nature as well as the phase matching properties. Herein, we report the anisotropic behavior of gamma glycine in mechanical and laser damage threshold properties and the dependence of particle size with respect to second harmonic generation. Apart from these studies, single and powder X-ray diffraction analyses, UV-Vis spectroscopy, FT-IR analysis, solubility and morphology study, dielectric and thermal studies were carried out to exploit the feasibility of the title compound for NLO applications.

\section{Experimental}

\subsection{Material synthesis}

The starting materials were lithium nitrate (AR grade: Hi-media) and glycine (AR grade:
Qualigens) taken in the 1:2 ratio. At first, glycine and lithium nitrate were dissolved separately in double distilled water and stirred continuously using immersible magnetic stirrer until a clear solution appeared, at room temperature. After this, lithium nitrate solution was added to the glycine solution with continuous stirring until the both solutions were mixed well and the resulting solution was allowed to mix well for the time of $5 \mathrm{~h}$. The resultant solution was filtered using Whatman filter paper. The prepared solution was allowed to evaporate at room temperature and the synthesized salt was subjected to repeated recrystallization process several times for its purity.

\subsection{Crystal growth}

Single crystal of GG was grown by slow evaporation technique. The recrystallized salt of GG was taken as a raw material. The saturated solution of $\gamma$-glycine was prepared at room temperature (ca. $30{ }^{\circ} \mathrm{C}$ ) using double distilled water as a solvent. The prepared solution was filtered using Whatman filter paper. The beaker containing the solution was closed with a perforated cover and kept in a dust free atmosphere. After attainment of supersaturation by solvent evaporation, tiny crystals were formed. Optically transparent good quality single crystal of $\gamma$-glycine was harvested after a period of 25 days and is shown in Fig. 1.

\section{Characterization studies}

Single crystal X-ray diffraction (SXRD) analysis was carried out for GG crystal, using ENRAF NONIUS CAD4 automatic X-ray diffractometer with $\operatorname{MoK} \alpha(\lambda=0.7170 \AA)$ radiation to study its unit cell dimensions and morphology. The powder $\mathrm{X}$-ray diffraction analysis was carried out in order to reveal the crystalline nature of GG using ReichSeifert diffractometer with $\mathrm{CuK} \alpha(\lambda=1.5418 \AA)$ radiation in the range of $10^{\circ}$ to $70^{\circ}$ at a scan rate of $2 \% \mathrm{~min}$. The grown $\gamma$-glycine was subjected to FT-IR analysis to confirm the presence of functional groups by employing PERKIN-ELMER spectrometer with a resolution of $1.0 \mathrm{~cm}^{-1}$. The UV-Vis transmittance spectrum for a $\gamma$-glycine single crystal was recorded in the wavelength 


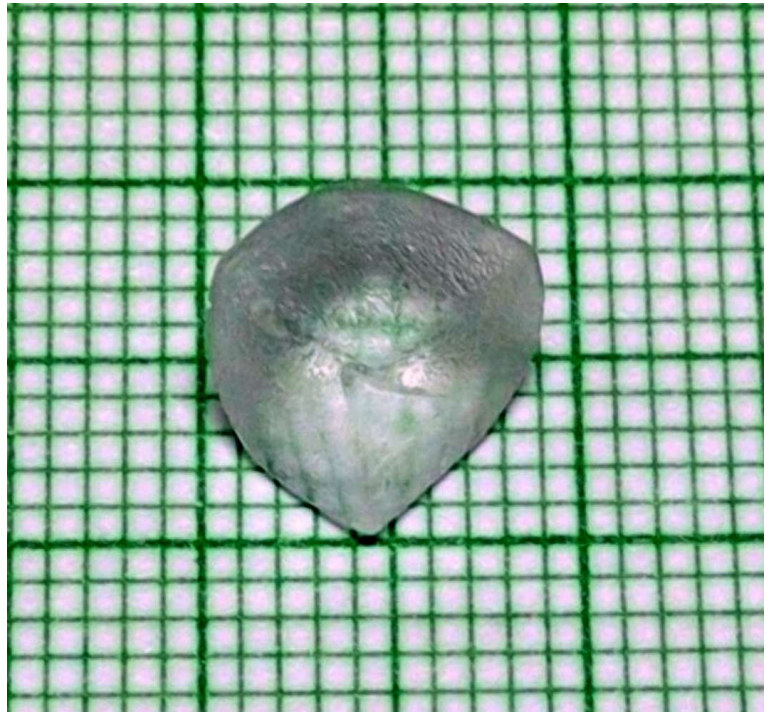

Fig. 1. As-grown $\gamma$-glycine single crystal.

of $200 \mathrm{~nm}$ to $800 \mathrm{~nm}$ to determine its optical transmittance range using VARIAN CARY 5E UV-Vis spectrophotometer. Thermogravimetric and differential thermal analysis (TG-DTA) was carried out to investigate the thermal stability of the crystal, using NETZSCH STA 409 PC/PG thermal analyzer in nitrogen atmosphere in the temperature range of $30{ }^{\circ} \mathrm{C}$ to $500{ }^{\circ} \mathrm{C}$. Microhardness measurement was carried out on the prominent planes at room temperature using Shimadzu HMV-2000 fitted with Vickers pyramidal indentor to evaluate the mechanical stability. The dielectric measurement was carried out using HIOKI 3532 LCR HITESTER. Laser damage threshold investigation was carried out on the prominent planes using Nd: YAG laser to study the anisotropic nature of the title material. The existence of second harmonic generation was confirmed by Kurtz-Perry powder technique.

\section{Results and discussion}

\subsection{Single crystal $X$-ray diffraction and powder $\mathrm{X}$-ray diffraction analyses}

Gamma-glycine single crystal was subjected to single crystal X-ray diffraction analysis (SXRD) using ENRAF NONIUS CAD4 automatic $\mathrm{X}$-ray diffractometer with $\operatorname{MoK} \alpha(\lambda=0.7170$ $\AA)$ radiation. The cell parameters of $\gamma$-glycine single crystal obtained from SXRD study are as follows, $a=7.02$ (12) $\AA, b=7.02$ (12) $\AA$, $\mathrm{c}=5.47$ (9) $\AA, \alpha=\beta=90^{\circ}, \gamma=120^{\circ}$, and $\mathrm{V}=233$ (4) $\AA^{3}$. The $\gamma$-glycine single crystal belongs to a hexagonal crystal system with noncentrosymmetric space group $\mathrm{P} 3_{1}$ which are the necessary criteria for SHG. These values are found to be in good agreement with the reported data [7]. The powder X-ray diffraction analysis was carried out in order to reveal the crystalline nature of GG using Reich-Seifert diffractometer with $\mathrm{CuK} \alpha$ $(\lambda=1.5418 \AA)$ radiation in the range of $10^{\circ}$ to $70^{\circ}$ at the scan rate of $2 \% \mathrm{~min}$. From the obtained powder X-ray diffractogram, various planes of reflection were indexed using POWDERX program. The indexed powder X-ray diffraction pattern for $\gamma$-glycine powdered crystalline sample is shown in Fig. 2.

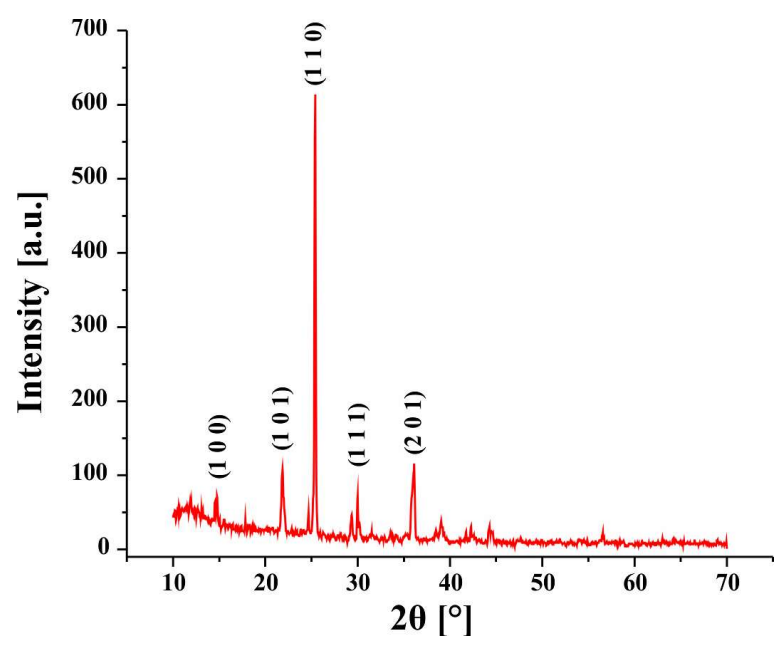

Fig. 2. Powder X-ray diffractogram of $\gamma$-glycine crystalline sample.

From the PXRD analysis it is confirmed that $\gamma$-glycine has high crystalline nature. The powder $\mathrm{X}$-ray diffractogram of $\gamma$-glycine from our experimental work has been compared with the simulated powder X-ray pattern of $\gamma$-glycine crystals from the corresponding CIF files using MERCURY software as shown in Fig. 3. It is clear from Fig. 3 that the simulated PXRD pattern of $\gamma$-glycine agrees well with the experimental PXRD pattern of $\gamma$-glycine with the dominant plane of $\left(\begin{array}{lll}1 & 1 & 0\end{array}\right)$ at $25^{\circ}$

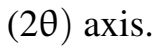




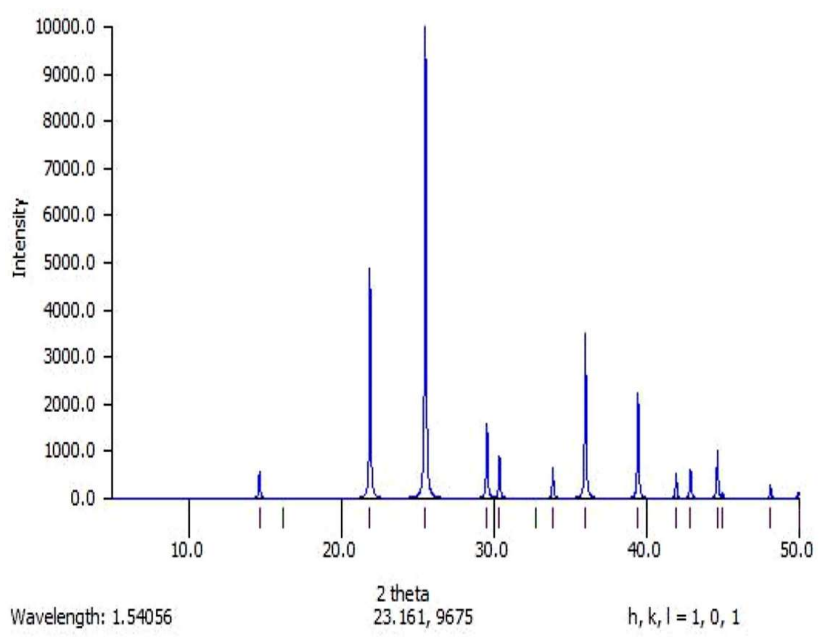

(a)

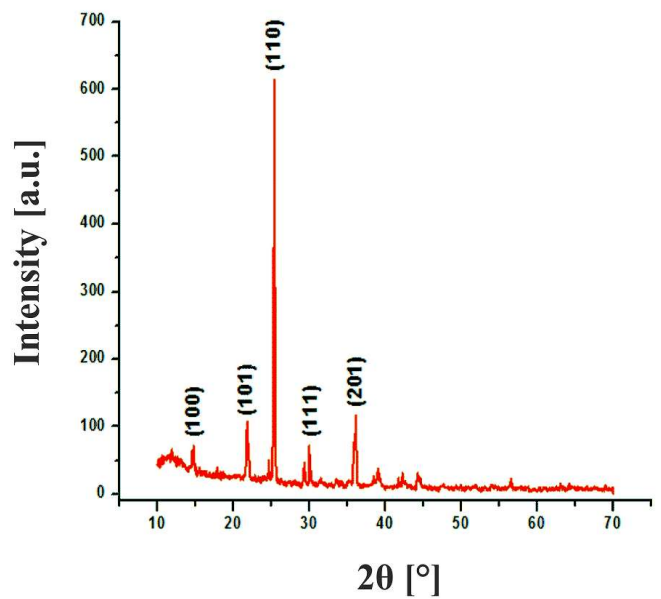

(b)

Fig. 3. Comparison of powder X-ray diffractograms: (a) simulated PXRD pattern of $\gamma$-glycine and (b) experimental PXRD pattern of $\gamma$-glycine.

\subsection{Prediction of crystal morphology}

The morphology of the grown single crystal of $\gamma$-glycine polymorph was investigated by identifying various growth faces by the goniometric method using ENRAF NONIUS CAD4 automatic X-ray diffractometer with $\operatorname{MoK} \alpha(\lambda=0.7170 \AA)$ radiation and its schematic sketch is depicted in Fig. 4. As-grown crystal of dimensions $10 \mathrm{~mm} \times 8 \mathrm{~mm} \times 8 \mathrm{~mm}$ was used for the study. This study is useful for understanding the growth rate anisotropy of the grown crystals. Single crystal of $\gamma$-glycine consists of six well developed faces out of which the planes $\left(\begin{array}{lll}0 & 1 & 1\end{array}\right),\left(\begin{array}{lll}-1 & 0 & 1\end{array}\right)$ and $(1-11)$ are prominent. The $\gamma$-glycine single crystal has a trigonal end with smooth facets and truncated rough facets at the opposite end. It has been found that the growth rates of GG crystal along a and $c$ axes are lesser than that along $b$ axis.

\subsection{Spectral analysis}

Vibrational spectroscopy (FT-IR) is an extremely useful tool for examination of molecular structure of chemical compounds. All chemical compounds have their own typical IR spectrum. The grown $\gamma$-glycine was subjected to FT-IR analysis to confirm the presence of functional groups by employing PERKIN-ELMER spectrometer in
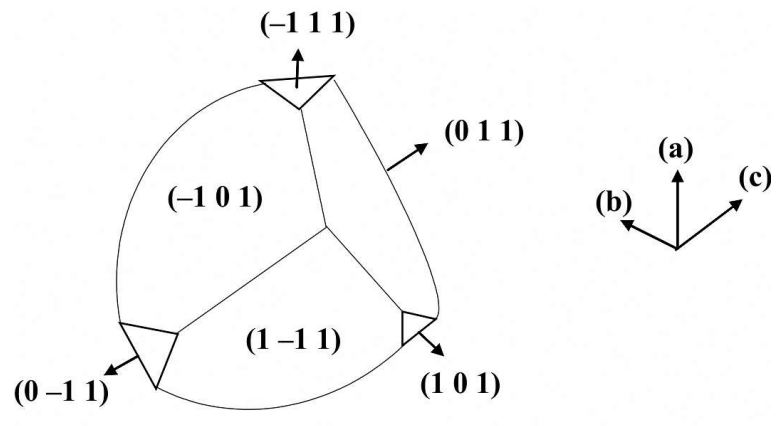

Fig. 4. Morphology of $\gamma$-glycine single crystal.

the range of $4000 \mathrm{~cm}^{-1}$ to $400 \mathrm{~cm}^{-1}$ using the $\mathrm{KBr}$ pellet technique with a resolution of $1.0 \mathrm{~cm}^{-1}$. The FT-IR spectrum of $\gamma$-glycine is shown in Fig. 5. The bands arising due to $\mathrm{CH}_{2}$ and $\mathrm{NH}_{3}^{+}$ stretching vibrations are found to occur at high wave number region of $2167 \mathrm{~cm}^{-1}$ to $2598 \mathrm{~cm}^{-1}$ and $2794 \mathrm{~cm}^{-1}$ to $3441 \mathrm{~cm}^{-1}$, respectively. The peak around $1489 \mathrm{~cm}^{-1}$ is attributed to symmetric stretching mode of $\mathrm{COO}^{-}$group. The peak at $1323 \mathrm{~cm}^{-1}$ is assigned to $\mathrm{CH}_{2}$ wagging vibrations [8]. The peak at $929 \mathrm{~cm}^{-1}$ is due to the bending vibration of $\mathrm{C}-\mathrm{H}$ group. The peaks due to $\mathrm{C}-\mathrm{N}$ stretching vibrations and $\mathrm{C}-\mathrm{C}$ stretching vibrations are found to occur at $1043 \mathrm{~cm}^{-1}$ and $889 \mathrm{~cm}^{-1}$, respectively. The bands arising as a result 
of carboxylate group are observed at $501 \mathrm{~cm}^{-1}$, $607 \mathrm{~cm}^{-1}$ and $686 \mathrm{~cm}^{-1}$, while the peak observed at $1126 \mathrm{~cm}^{-1}$ is attributed to $\mathrm{NH}_{3}^{+}$group. The presence of both the vibrations of carboxylate and ammonium ion clearly indicates that the glycine molecule exists in zwitterionic form in $\gamma$-glycine.

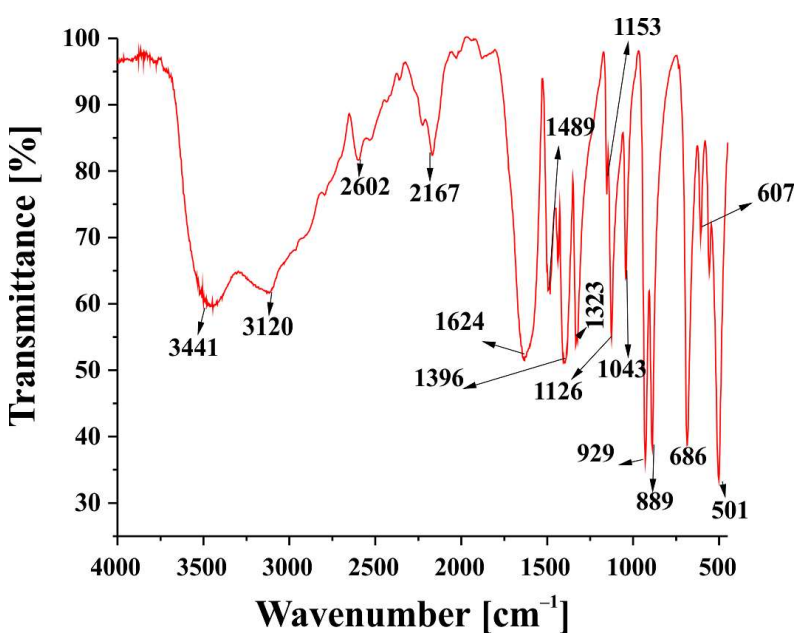

Fig. 5. FT-IR spectrum of $\gamma$-glycine.

\subsection{UV-Vis spectral analysis}

The UV-Vis spectral analysis of $\gamma$-glycine single crystal was recorded in the range of $200 \mathrm{~nm}$ to $800 \mathrm{~nm}$ using a polished crystal sample of 9 $\mathrm{mm}$ in length and $2 \mathrm{~mm}$ in thickness. The recorded optical transmittance spectrum is shown in Fig. 6. GG single crystals are found to have high transmittance of about $80 \%$ with the cut-off wavelength of $299 \mathrm{~nm}$, thereby confirming that the crystal has a wider transparency range indicating that the grown crystal is a promising candidate for NLO applications. The cut-off of $299 \mathrm{~nm}$ for the title compound is due to the $\mathrm{n} \rightarrow \pi *$ transitions. This result is in good agreement with reported data [9].

The obtained transmittance was used to calculate the absorption coefficient $(\alpha)$ using the relation:

$$
\alpha=\frac{2.3026}{t} \log \left(\frac{1}{T}\right)
$$

where $\mathrm{T}$ is the transmittance and $\mathrm{t}$ is the thickness of the crystal sample.

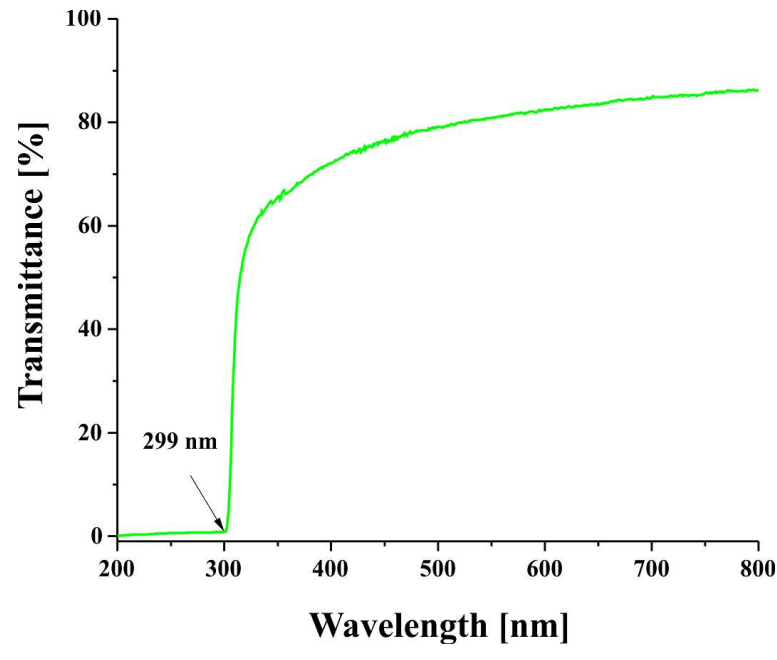

Fig. 6. Transmittance spectrum of $\gamma$-glycine crystal.

The energy dependence of absorption coefficient in the high photon energy region suggests the occurrence of direct band gap of the crystal obeying the following equation for high photon energies (hv) [10]:

$$
(\alpha h v)^{2}=A\left(E_{g}-h v\right)
$$

where, $\mathrm{h}$ is the Planck's constant, $\mathrm{A}$ is a constant, $v$ is the frequency of incident photon and $E_{g}$ is the optical bandgap.

The band gap of the crystal was evaluated by extrapolating the linear part of the graph to the energy axis and was found to be $4.06 \mathrm{eV}$ using Tauc's plot [11] between $(\alpha \mathrm{h} v)^{2}$ and the photon energy (hv) which is shown in Fig. 7. The wide band gap of GG crystal confirms the large transmittance in the visible region.

\subsection{Thermal properties}

The thermal behavior of GG crystalline sample was studied by thermogravimetric (TG) analysis and differential thermal analysis (DTA) using GG crystalline sample of $4.612 \mathrm{mg}$. A thermal analyzer was employed at a heating rate of $10{ }^{\circ} \mathrm{C} / \mathrm{min}$ in nitrogen atmosphere. The TG and DTA thermograms were recorded in the temperature range of $30{ }^{\circ} \mathrm{C}$ to $500{ }^{\circ} \mathrm{C}$. The recorded thermograms of $\alpha$ - and $\gamma$-glycine polymorphs are shown in Fig. 8 and Fig. 9, respectively. 


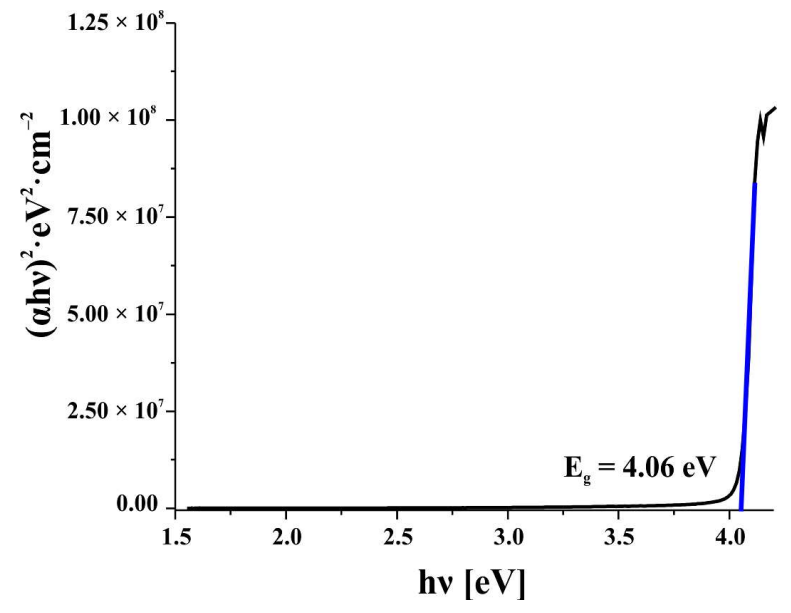

Fig. 7. Tauc's plot of $(\alpha h v)^{2}$ vs. photon energy of $\gamma$-glycine crystal.

The TG-DTA curve of commercially available $\alpha$-glycine does not show any variation in the heat flow until the melting point that occurs at $251{ }^{\circ} \mathrm{C}$. The melting transition actually starts at around $230{ }^{\circ} \mathrm{C}$ and ends at $251{ }^{\circ} \mathrm{C}$. Also, no appreciable mass loss was observed until the melting point. At ambient temperature $\gamma$-glycine is thermodynamically most stable polymorphic form. But it changes into $\alpha$-form upon heating at about $165{ }^{\circ} \mathrm{C}$ [7]. The TG-DTA curve of $\gamma$-glycine shows an endothermic peak at $168{ }^{\circ} \mathrm{C}$ before its melting point. This peak represents certainly a phase transformation of $\gamma$-form probably to $\alpha$-form. Except this, there is no big change in the thermal behavior of this crystal until its melting transition that occurs at $251{ }^{\circ} \mathrm{C}$. Hence, $\gamma$-glycine is thermally stable up to $251{ }^{\circ} \mathrm{C}$ and may be designed for NLO application up to $251{ }^{\circ} \mathrm{C}$ taking into account the phase transition temperature of $168^{\circ} \mathrm{C}$.

\subsection{Microhardness properties}

Microhardness study was carried out on GG single crystal using a Vickers microhardness tester fitted with a Vickers diamond pyramidal indenter in order to evaluate crystal mechanical stability. The static indentations were made at room temperature at a constant indentation time of $5 \mathrm{~s}$ for all indentations. A cut and polished sample of $4 \mathrm{~mm}$ thickness was used for the study. The indentation marks were made on the prominent faces $\left(\begin{array}{lll}-1 & 0 & 1\end{array}\right),\left(\begin{array}{lll}1 & -1 & 1\end{array}\right)$

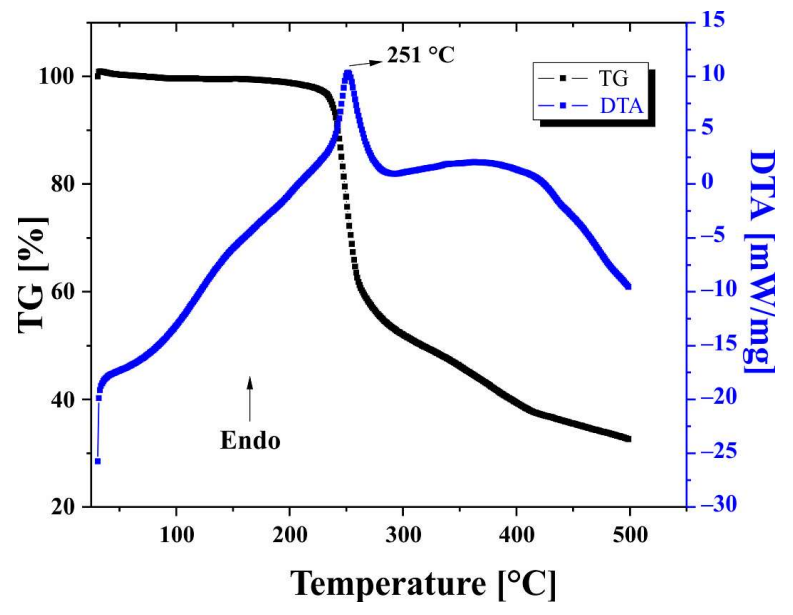

Fig. 8. TG-DTA thermogram of $\alpha$-glycine.

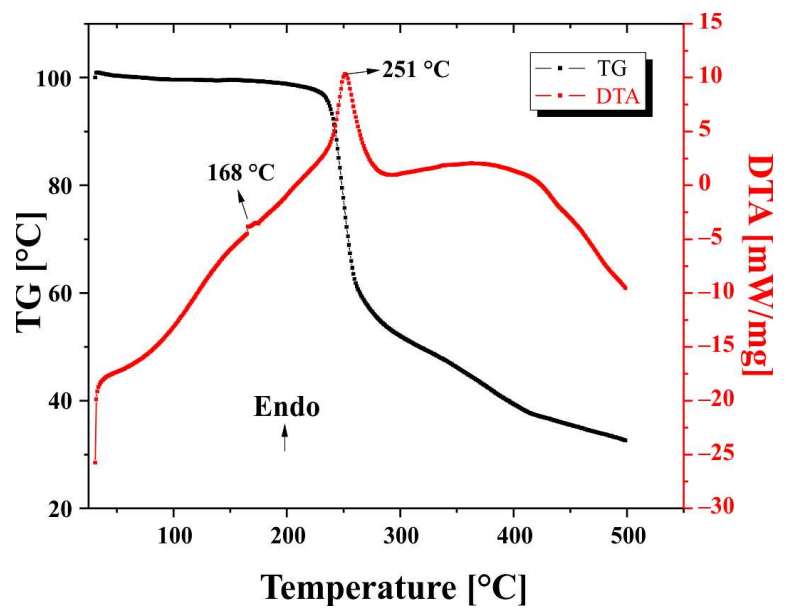

Fig. 9. TG-DTA thermogram of $\gamma$-glycine.

and $\left(\begin{array}{lll}0 & 1 & 1\end{array}\right)$ by varying the load from $10 \mathrm{~g}$ to $60 \mathrm{~g}$ to elucidate the anisotropic nature of hardness on these planes.

The Vickers microhardness number of the title crystal was calculated using the relation:

$$
H_{v}=1.8544\left(\frac{P}{d^{2}}\right)\left(\mathrm{kg} / \mathrm{mm}^{2}\right)
$$

where $\mathrm{H}_{\mathrm{v}}$ is the Vickers hardness number, $\mathrm{P}$ is the applied load and $\mathrm{d}$ is the average diagonal length of the indentation mark.

The plots between hardness number and load for GG single crystals on different planes, $\left(\begin{array}{lll}-1 & 0 & 1\end{array}\right)$, $\left(\begin{array}{lll}1 & -1 & 1\end{array}\right)$ and $\left(\begin{array}{lll}0 & 1 & 1\end{array}\right)$ planes are depicted in Fig. 10. 
It is clear that the microhardness number increases with increasing load.

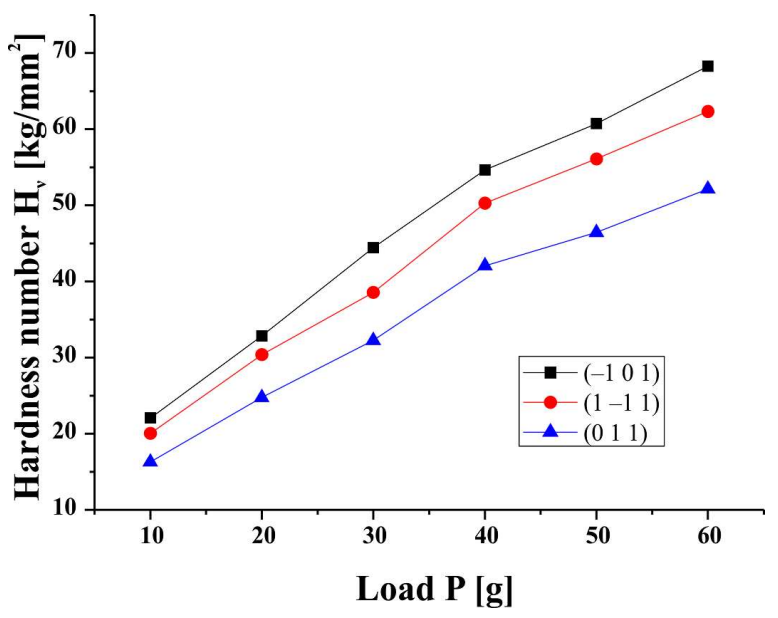

Fig. 10. Microhardness number vs. load for GG (a) $\left(\begin{array}{lll}-1 & 0 & 1\end{array}\right)$ plane, (b) $\left(\begin{array}{lll}1 & -1 & 1\end{array}\right)$ plane and (c) (llll) plane.

It is evident from the above plot that the microhardness value of the crystal increases with an increase in load which is in agreement with the reverse indentation size effect (ISE) [12].

Hardness values of $\gamma$-glycine crystal differ from one plane to another, which confirms the microhardness anisotropy. For example, it is clear from Fig. 8 that the maximum hardness numbers observed on (-1 $\left.\begin{array}{lll}-1 & 0\end{array}\right),\left(\begin{array}{lll}1 & -1 & 1\end{array}\right)$ and $\left(\begin{array}{lll}0 & 1 & 1\end{array}\right)$ planes of $\gamma$-glycine crystal are $68.25 \mathrm{~kg} / \mathrm{mm}^{2}, 62.32 \mathrm{~kg} / \mathrm{mm}^{2}$ and $52.14 \mathrm{~kg} / \mathrm{mm}^{2}$, respectively, at the load of $60 \mathrm{~g}$. Above $60 \mathrm{~g}$ load, cracks are developing due to the release of internal stress generated locally by indentation and hence, the hardness value decreases. The lower hardness value is due to the presence of relatively weak bonds in GG.

The ratio of differences in maximum hardness of two different planes to the maximum value of microhardness of the crystal gives the anisotropy coefficient (A), which is given by the relation [13]:

$$
A=\frac{\Delta H_{v}}{H_{v}}
$$

The reason for this nonlinear behavior of the microhardness of the crystal along different planes may be due to different bond strength of the crystal in different planes and cleavage plane of the samples.

\subsection{Dielectric studies}

The dielectric constant and dielectric loss of the grown crystals were studied at different temperatures using HIOKI 3532 LCR HITESTER in the frequency region of $100 \mathrm{~Hz}$ to $5 \mathrm{MHz}$. A sample of uniform cross-sectional area cut along the growth axis was polished and placed between the two copper electrodes which acted as a parallel plate capacitor. Silver paint was coated on the surface of the sample in order to make firm electrical contact.

The capacitance and dielectric loss of the sample were measured at various frequencies, in the range of $100 \mathrm{~Hz}$ to $5 \mathrm{MHz}$ at different temperatures. The dielectric constant was calculated using the relation $\epsilon_{\mathrm{r}}=\mathrm{Ct} / \epsilon_{0} \mathrm{~A}$, where $\mathrm{C}$ is capacitance, $\mathrm{t}$ is thickness of the sample, $\epsilon_{0}$ is permittivity of free space and $A$ is area of cross section of a sample. The observations were made while cooling the sample. The plot of dielectric constant $\left(\epsilon_{\mathrm{r}}\right)$ and dielectric loss $(\tan \delta)$ versus applied frequency for $\gamma$-glycine crystal are shown in Fig. 11 and Fig. 12, respectively.

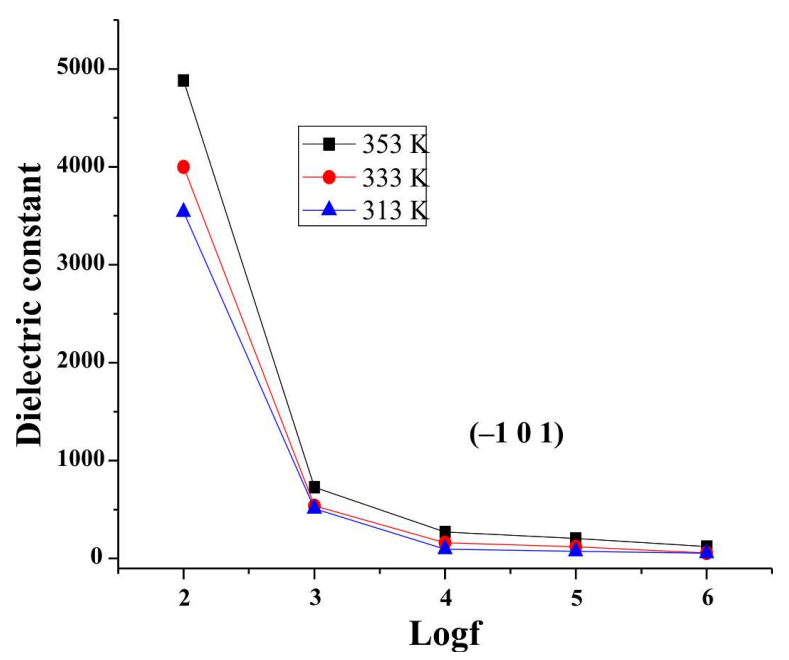

Fig. 11. Plot of dielectric constant vs. log frequency.

Typical sample dimensions of $\gamma$-glycine crystal were $8.68 \mathrm{~mm}^{2} \times 4.42 \mathrm{~mm}^{2}$ in surface area 


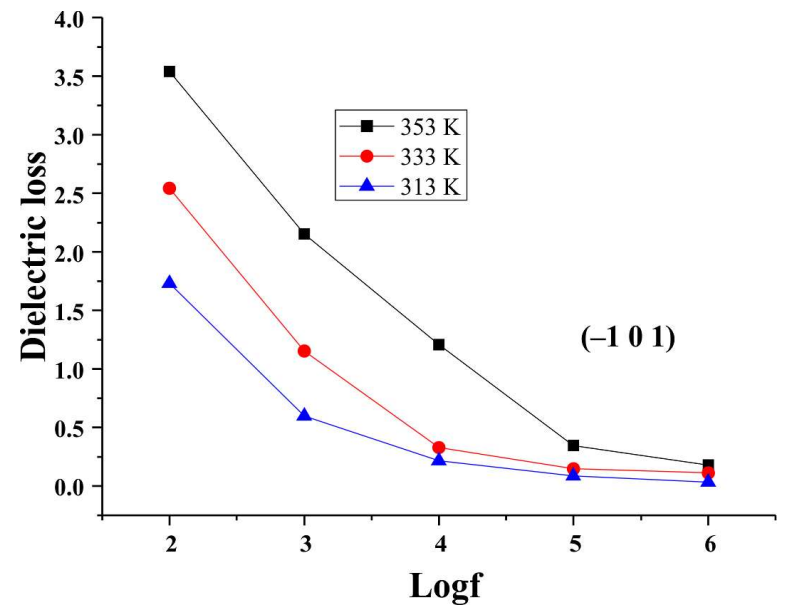

Fig. 12. Plot of dielectric loss vs. log frequency.

and $2.04 \mathrm{~mm}$ in thickness. The dielectric constant measurement for $\gamma$-glycine crystal was carried out on their prominent $\left(\begin{array}{lll}-1 & 0 & 1\end{array}\right)$ plane. The dielectric constant has high values in the low frequency region and then it decreases with further increase of applied frequency. The dielectric constant decreases very rapidly at low frequencies and slowly at higher frequencies. The electronic exchange between the ions in the crystal causes local displacement of electrons in the direction of the applied field that results in polarization. This is a normal dielectric behavior that both dielectric constant and dielectric loss decrease with an increase in frequency. The very high value of $\epsilon_{\mathrm{r}}$ at lower frequencies may be attributed to the presence of all four polarizations, namely space charge, orientational, electronic and ionic polarizations and its low value at higher frequencies may be due to the gradual loss of significance of these polarizations.

The space charge contribution depends on the purity and perfection of the material and it has a noticeable influence in the low frequency region. The higher values of dielectric constant at lower frequencies may be attributed to space charge polarization and also to crystal lattice defects. As the frequency increases, a point is reached where the space charge cannot sustain and comply with the external field, hence, polarization decreases with frequency giving rise to diminishing the value of $\epsilon_{\mathrm{r}}$ and dielectric loss $(\tan \delta)$. Continuous gradual decrease in dielectric loss $(\tan \delta)$ as well as $\epsilon_{\mathrm{r}}$ suggests that the crystals, similar to any normal dielectrics, may have domains of different sizes and varying relaxation times. In accordance with Miller rule, the lower value of dielectric constant is a suitable parameter for the enhancement of SHG efficiency [14]. The low value of dielectric loss $(\tan \delta)$ in the region of high frequency for the sample suggests that the grown crystals possess enhanced optical quality with lesser number of electrically active defects and this parameter is of vital importance for non-linear optical materials in their applications [15].

\subsection{Laser damage threshold}

The mechanism of laser induced damage in materials is often very complex involving various processes, like electron avalanche, multi photon absorption, photo ionization by thermal absorption, photochemical dislocations, etc. [16]. The optically good quality cut and polished flat crystal sample of dimension $10 \mathrm{~mm} \times 5 \mathrm{~mm} \times 4 \mathrm{~mm}$ was mounted on the crystal holder in the path of the Nd: YAG laser beam of fundamental wavelength $1064 \mathrm{~nm}$ and pulse width of $8 \mathrm{~ns}$. The laser beam with a radius of $1 \mathrm{~mm}$ was focused on the crystal. The energy of the beam was increased from $5 \mathrm{~mJ}$ until damage was observed. The laser damage threshold of the crystal was calculated using the expression:

$$
\text { Power density }=E / \tau \pi r^{2}\left(\mathrm{GW} / \mathrm{cm}^{2}\right)
$$

where $E$ is the input energy $(\mathrm{mJ}), \tau$ is the pulse width (ns), $r$ is the radius of the circular spot $(\mathrm{mm})$.

The multiple shots (10 pulses) laser damage threshold (LDT) studies were carried out on the prominent planes $\left(\begin{array}{llll}-1 & 0 & 1\end{array}\right),\left(\begin{array}{lll}1 & -1 & 1\end{array}\right)$ and $\left(\begin{array}{lll}0 & 1 & 1\end{array}\right)$ and the values were determined as $0.88 \mathrm{GW} / \mathrm{cm}^{2}$, $0.68 \mathrm{GW} / \mathrm{cm}^{2}$ and $0.52 \mathrm{GW} / \mathrm{cm}^{2}$, respectively. The single shot (1 pulse) laser damage threshold values were determined as $1.32 \mathrm{GW} / \mathrm{cm}^{2}$ for $\left(\begin{array}{lll}-1 & 0 & 1\end{array}\right)$, $0.94 \mathrm{GW} / \mathrm{cm}^{2}$ for $(1-11)$ and $0.79 \mathrm{GW} / \mathrm{cm}^{2}$ for $\left(\begin{array}{lll}0 & 1 & 1\end{array}\right)$ planes, respectively. They were found to be higher than that of the standard nonlinear optical KDP $\left(0.2 \mathrm{GW} / \mathrm{cm}^{2}\right)$ crystals [16]. The variations in the LDT values on different planes may be attributed to the anisotropic nature of the material in question. The careful examination 
of damaged crystal through optical microscope gave some idea about the mechanism underlying in the laser induced damage [17]. The microscopic image of laser damage threshold pattern of gamma glycine crystal on $\left(\begin{array}{lll}-1 & 0 & 1\end{array}\right)$ plane for multiple shot and single shot is shown in Fig. 13. Only the $\left(\begin{array}{lll}-1 & 0 & 1\end{array}\right)$ plane is considered because of its high LDT values, while comparing it with other planes. It shows circular blobs surrounding the core of the damage. Such circular blobs are generally seen in crystals where the damage is mainly due to thermal effects resulting in melting and solidification or decomposition of the material [18]. It is also evident from the earlier report [19], that in the long-pulse regime ( $\tau>100 \mathrm{ps}$ ), the damage is controlled by the rate of thermal conduction through the atomic lattice and in the short-pulse ( $\tau<100 \mathrm{ps})$, it is due to optical breakdown and various nonlinear ionization mechanisms. Hence, thermal effect becomes important for the pulse width that is of nanosecond and longer. The local heating caused by macroscopic inclusion is also a more severe problem in the high intensity short-pulse system than in lowintensity long-pulse. In the present work, we have used $8 \mathrm{~ns}$ pulse.
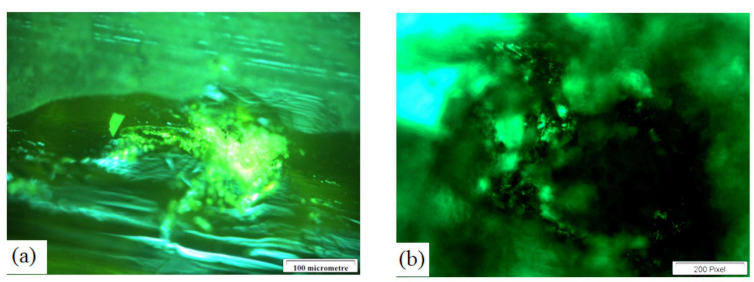

Fig. 13. (a) multiple shot Laser damage pattern of $\gamma$ glycine for $\left(\begin{array}{lll}-1 & 0 & 1\end{array}\right)$ plane and (b) single shot laser damage pattern of $\gamma$-glycine for $\left(\begin{array}{lll}-1 & 0 & 1\end{array}\right)$ plane.

\subsection{Nonlinear optical study}

NLO materials have attracted increasing attention due to their wide applications in the recent technologies like lasers, optical communications and data storage [20]. The SHG effective nonlinearity of $\gamma$-glycine powdered sample was determined using Kurtz and Perry powder technique [21]. This technique enables measuring the SHG effective nonlinearity of NLO materials relative to standard potassium dihydrogen phosphate (KDP). A Q-switched Nd: YAG laser operating at $1064 \mathrm{~nm}$ and $8 \mathrm{~ns}$ pulse width with an input repetition rate of $10 \mathrm{~Hz}$ and energy $5 \mathrm{~mJ} /$ pulse was used for this study. The grown single crystal of $\gamma$-glycine was crushed into powder with a uniform particle size and then packed into a microcapillary tube with a uniform pore size and exposed to laser radiation. The SHG output was converted into electrical signal and was displayed on a digital storage oscilloscope. The optical signal incident on photo multiplier tube was converted into voltage output. The second harmonic signal generated in the crystalline sample was confirmed from the emission of green radiation of wavelength $532 \mathrm{~nm}$ from the crystalline powder. The second harmonic signal of $27.8 \mathrm{mV}$ was obtained for $\gamma$-glycine crystal, while the standard potassium dihydrogen phosphate (KDP) crystal gives a SHG signal of $13.4 \mathrm{mV}$ for the same input energy. It shows the SHG effective nonlinearity of $\gamma$-glycine is about 2 times larger than that of standard NLO material KDP. This value of SHG nonlinearity agrees well with the reported values [2] of $\gamma$-glycine grown from lithium nitrate as an additive.

\subsection{Particle size-dependence of SHG}

Particle size dependence of SHG is used as a screening methodology to identify materials with a capability of phase matching. The continuous increase of SHG signal with an increase of particle size and its saturation confirms the phase matching behavior of the material. To confirm the existence of phase matching property, the particle size dependence of SHG intensity was studied. The SHG nonlinearity have been shown to depend strongly on particle size [22]. Only the materials exhibiting a phase matching behavior are grown into single crystals of large size for NLO applications [23]. The particle size dependence of SHG was carried out by sieving the $\gamma$-glycine powdered crystalline sample into various microcapillary tubes of sieve sizes ranging from $55 \mu \mathrm{m}$ to $400 \mu \mathrm{m}$. Measuring SHG as a function of particle size provides clear information about type of phase matching condition. 
If type-1 phase-matching occurs, the SHG nonlinearity value increases with the particle size and saturates at a maximum value. If phase- 1 matching does not occur, the SHG nonlinearity reaches a maximum value and then decreases, as the particle size increases [24]. Single crystal of $\gamma$-glycine was ground and sieved into distinct particle size ranges, below $55 \mu \mathrm{m}, 55 \mu \mathrm{m}$ to $125 \mu \mathrm{m}, 125 \mu \mathrm{m}$ to $250 \mu \mathrm{m}, 250 \mu \mathrm{m}$ to $400 \mu \mathrm{m}$, and above $400 \mu \mathrm{m}$. A Q-switched Nd: YAG laser operating at $1064 \mathrm{~nm}$ and $8 \mathrm{~ns}$ pulse width with an input repetition rate of $10 \mathrm{~Hz}$ and energy $4.7 \mathrm{~mJ} /$ pulse was used for this study. The SHG output signal voltage increased almost linearly with an increase in particle size up to $125 \mu \mathrm{m}$ to $250 \mu \mathrm{m}$. The SHG output voltage deviated from the linearity above $250 \mu \mathrm{m}$ and achieved saturation. The particle size dependency of SHG output for GG crystals is depicted in Fig. 14. It is evident from Fig. 14 that $\gamma$-glycine exhibits type- 1 phase matching as the SHG nonlinearity increases with the particle size and saturates at a maximum value. This kind of particle size dependence of SHG intensity was also observed in phase matchable crystals [25]. Therefore $\gamma$-glycine crystal can be used as an efficient candidate for frequency doublers and optical parametric oscillators.

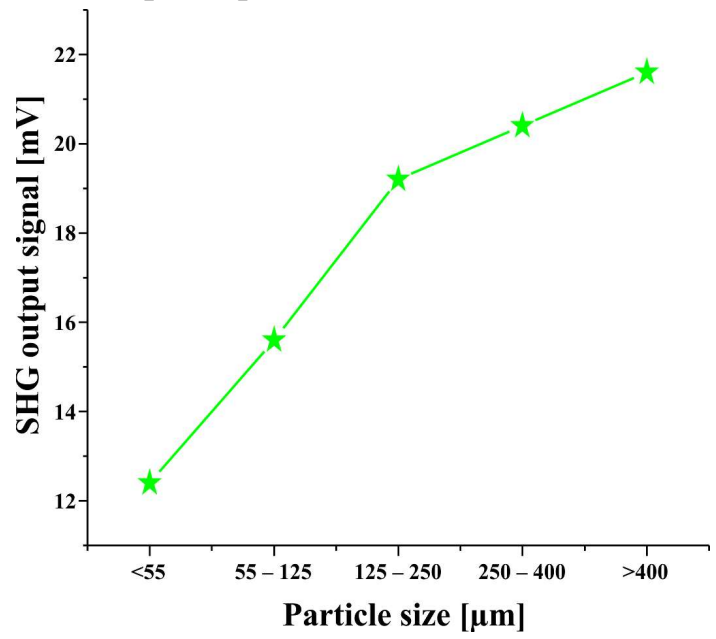

Fig. 14. Particle size vs. SHG output signal.

\section{Conclusions}

Optically good quality polymorph $\gamma$-glycine single crystal was grown by slow evaporation technique using lithium nitrate as an additive. The cell parameters were identified using single crystal X-ray diffraction analysis. The crystalline nature was analyzed using powder X-ray diffraction analysis. The morphological analysis was carried out in order to throw light on the growth rate anisotropy and to identify various facets. The spectral analysis was done to confirm the presence of various expected functional groups. From the UV-Vis analysis it was confirmed that $\gamma$-glycine crystals have a wide transparency in the range of $299 \mathrm{~nm}$ to $800 \mathrm{~nm}$. The band gap was evaluated from the transmittance spectrum. The thermal behavior of the title material was studied from TG-DTA analysis. Vickers microhardness study was carried out to evaluate the anisotropic and mechanical behavior of $\gamma$-glycine. The electrical behavior was analyzed by dielectric measurement. The laser damage threshold study was carried out for $\gamma$-glycine single crystals using single and multiple shots for evaluating the laser stability for NLO applications. The relative SHG nonlinearity was assessed and the existence of SHG was confirmed using Kurtz-Perry powder technique. The particle size dependence of SHG was done to have a clear picture about the type of phase matching. The promising crystal growth and physicochemical properties of $\gamma$-glycine single crystal nominate it as a potential material for photonics, electro-optic and SHG device applications.

\section{References}

[1] PRasad P.N., Williams D.J., Introduction to Nonlinear Optical Effects in Molecules and Polymers, John Wiley \& Sons, Inc. New York, 1991.

[2] Ashokkumar R., Ezhilvizhi R., ViJayan N., RAJANBABU D., Physica B, 406 (2011), 2594.

[3] Dillip G.R., Raghavaiah P., Mallikarjuna K., MADHUKARREDDY C., BhagaVANNARAYANA G., Rameshiumar V., Devaprasadraju B., Spectrochim. Acta A, 79 (2011), 1123.

[4] Parimaladevi R., SeKar C., Spectrochim. Acta A, 76 (2010), 490.

[5] Dhanaraj P.V., Rajesh N.P., Mater. Chem. Phys., 115 (2009), 413.

[6] Esthakupeter M., Ramasamy P., Spectrochim. Acta A, 75 (2010), 1417.

[7] LitAKA Y., Acta Crystallogr., 14 (1961), 1.

[8] Silverstein R.M., Bassler G.C., Morrill T.C., Spectrometric Identification of Organic Compounds, John Wiley \& Sons, New York, 1981. 
[9] Sekar C., Parimaladevi R., Spectrochim. Acta A, 74 (2009), 1160.

[10] Ashour A., El-Kadry N., Mahmoud S.A., Thin Solid Films, 269 (1995), 117.

[11] Tauc J., Grigorovici R., Vancu A., Phys. Status Solidi B, 15 (1966), 627.

[12] Sangwal K., Cryst. Res. Technol., 44 (2009), 1019.

[13] Bhaskaran A., Ragavan C.M., Sankar R., MoHANkUMaR R., Jayavel R., Cryst. Res. Technol., 42 (2007), 477.

[14] Debrus S., Ratajczak H., Venturini J., PinCON N., BARAN J., BARYCKI J., GLOWIAK T., PietraszKo A., Synthetic Met., 127 (2002), 99.

[15] RaK M., EREmin N.N., Eremina T.A., KuZnetsov V.A., OHKRIMENKo T.M., Furmanova N.G., EFREMOVA E.P., J. Cryst. Growth, 273 (2005), 577.

[16] ViJayan N., Bhagavannarayana G., GopalakrISHnan R., RAMASAMY P., Indian J. Chem. A, 46 (2007), 70.

[17] Yoshimura M., Kamimura T., Murase K., Mori Y., Yoshida H., NAKATSUKa M., SASAKI T., Jpn. J. Appl. Phys., 38 (1999), 129.
[18] Glass A.J., Guenther A.H., Appl. Optics, 12 (1973), 637.

[19] Stuart C., Feit M.D., Rubenchik A.M., Shore B.W., Perry M.D., Phys. Rev. Lett., 74 (1995), 2248.

[20] Russelraj K., Murugakoothan P., Mod. Phys. Lett. B., 26 (2012), 1150038.

[21] Kurtz S.K., Perry T.T., J. Appl. Phys., 39 (1968), 3798.

[22] Dougherty J.P., Kurtz S.K., J. Appl. Crystallogr., 9 (1976), 145.

[23] Porter Y., Kangmin O.K., Bhuvanesh N.S.P., Halas Yamani P.S., Chem. Mater., 13 (2001), 1910.

[24] Kangmin O.K., OKChi E., Halasyamani P.S., Chem. Soc. Rev., 35 (2006), 710.

[25] Wang L.N., Wang X.Q., Zhang G.H., LiU X.T., Sun Z.H., Sun G.H., J. Cryst. Growth, 327 (2011), 133.

Received 2016-07-07 Accepted 2017-01-19 